Suplemento alimentar à base de isolado proteico do soro de leite, probiótico e $\beta$-glucana: efeitos nas medidas antropométricas e imunidade em mulheres

Food supplement based on whey protein isolate, probiotic and $\beta$-glucan: effects on anthropometric measurements and immunity in women

Complemento alimenticio a base de aislado de proteína de suero, probiótico y $\beta$-glucano: efectos sobre las medidas antropométricas y la inmunidad en la mujer

Recebido: 11/12/2020 | Revisado: 17/12/2020 | Aceito: 18/12/2020 | Publicado: 21/12/2020

\title{
Orlando Barbieri Belloli
}

ORCID: https://orcid.org/0000-0001-5060-2024 Instituto Federal de Educação Ciência e Tecnologia do Rio Grande do Sul, Brasil E-mail: orlando.belloli@bento.ifrs.edu.br Joice Sifuentes dos Santos ORCID: https://orcid.org/0000-0002-1089-0620 Universidade Pitágoras Unopar, Brasil E-mail: Joice.sifuentes@gmail.com José Renato Silva ORCID: https://orcid.org/0000-0002-8289-7768 Universidade Pitágoras Unopar, Brasil E-mail: renato290@ hotmail.com Ivana Stefanini Carreiro Scachetti ORCID: https://orcid.org/0000-0003-4645-6380 Universidade Anhanguera de São Paulo, Brasil ivanastefanini@gmail.com Giselle Nobre Costa ORCID: https://orcid.org/0000-0001-6231-9445 Universidade Pitágoras Unopar, Brasil E-mail: gcnobre@gmail.com

Raul Jorge Hernan Castro-Gomez ORCID: https://orcid.org/0000-0001-5773-1654 Universidade Pitágoras Unopar, Brasil E-mail: rcastrog@yahoo.com 


\section{Resumo}

O mercado de alimentos com propriedades funcionais está em crescente expansão, e esse segmento está muito relacionado às inovações. Neste sentido, produtos contendo proteínas do soro do leite, popularmente conhecidas como "Whey Protein", ganham cada vez mais espaço no mercado de suplementos alimentares. O objetivo deste trabalho foi elaborar um suplemento alimentar a base de isolado protéico do soro de leite, probiótico Bifidobacterium lactis HN019 e $\beta$-glucana e avaliar os seus efeitos sobre os parâmetros antropométricos e níveis de imunoglobulina ( $\operatorname{Ig} \mathrm{A})$ salivar em mulheres praticantes de atividade física. O estudo foi realizado com 24 mulheres, idade média de 61 anos, com rotina de treino de15 minutos de alongamento e 45 minutos intercalados entre exercícios aeróbicos e resistidos. O suplemento alimentar foi administrado uma vez ao dia, por 90 dias. Foram feitas avaliações antropométricas (supra ilíaca, abdômen $(\mathrm{mm})$, tórax, cintura, quadril, circunferência abdominal (cm), \% de gordura, massa corporal magra e peso gordo) antes, durante e após a suplementação. A determinação de IgA salivar foi realizada nos mesmos períodos. Os resultados demonstraram que a suplementação aliada a uma rotina de exercícios físicos melhorou significativamente $(\mathrm{p}<0,05)$ as variáveis analisadas. Dentre as 9 variáveis antropométricas, 7 apresentaram diferença significativa $(\mathrm{p}<0,05)$. Os níveis de IgA apresentaram diferença significativa $(\mathrm{p}<0,05)$ entre as avaliações. Assim, o suplemento alimentar elaborado neste estudo, proporcionou melhoras significativas nos parâmetros avaliados, indicando o potencial do produto como promotor de efeitos benéficos no estado de saúde físico, bem como no estado imune do organismo.

Palavas-chave: Suplementação; Bifidobacterium lactis; Imunomodulação; Desenvolvimento de produtos.

\section{Abstract}

The food market with functional properties is growing, and this segment is closely related to innovations. In this sense, products containing whey proteins, popularly known as "Whey Protein", are gaining more and more space in the food supplement market. The objective of this work was to elaborate a food supplement based on whey protein isolate, probiotic Bifidobacterium lactis HN019 and $\beta$-glucan and to evaluate its effects on anthropometric parameters and levels of salivary immunoglobulin $(\operatorname{IgA})$ in women practicing physical activity. The study was carried out with 24 women, mean age 61 years, with a 15 -minute stretching routine and 45 minutes between aerobic and resistance exercises. The food supplement was administered once a day, for 90 days. Anthropometric assessments (supra 
iliac, abdomen (mm), chest, waist, hips, waist circumference $(\mathrm{cm}), \%$ fat, lean body mass and fat weight) were performed before, during and after supplementation. The determination of salivary IgA was performed in the same periods. The results showed that supplementation combined with a physical exercise routine significantly improved $(p<0.05)$ the analyzed variables. Among the 9 anthropometric variables, 7 showed a significant difference $(\mathrm{p}<0.05)$. IgA levels showed a significant difference $(p<0.05)$ between evaluations. Thus, the food supplement formulated in this study, provided significant improvements in the parameters evaluated, indicating the potential of the product as a promoter of beneficial effects on the physical health status, as well as on the body's immune status.

Keywords: Supplementation; Bifidobacterium lactis; Immunomodulation; Product development.

\section{Resumen}

El mercado de alimentos con propiedades funcionales está creciendo, y este segmento está muy relacionado con las innovaciones. En este sentido, los productos que contienen proteínas de suero, conocido popularmente como "Whey Protein”, están ganando cada vez más espacio en el mercado de complementos alimenticios. El objetivo de este trabajo fue elaborar un complemento alimenticio a base de aislado de proteína de suero, probiótico Bifidobacterium lactis HN019 y $\beta$-glucano y evaluar sus efectos sobre parámetros antropométricos y niveles de inmunoglobulina salival ( $\operatorname{IgA}$ ) en mujeres que practican actividad física. El estudio se realizó con 24 mujeres, edad media de 61 años, con una rutina de estiramiento de 15 minutos y 45 minutos entre ejercicios aeróbicos y de resistencia. El complemento alimenticio se administró una vez al día, durante 90 días. Se realizaron evaluaciones antropométricas (suprailíaca, abdomen $(\mathrm{mm})$, pecho, cintura, caderas, circunferencia de la cintura $(\mathrm{cm}), \%$ de grasa, masa corporal magra y peso graso) antes, durante y después de la suplementación. La determinación de IgA salival se realizó en los mismos períodos. Los resultados mostraron que la suplementación combinada con una rutina de ejercicio físico mejoró significativamente $(\mathrm{p}<0.05)$ las variables analizadas. Entre las 9 variables antropométricas, 7 mostraron una diferencia significativa $(\mathrm{p}<0,05)$. Los niveles de IgA mostraron una diferencia significativa $(p<0,05)$ entre las evaluaciones. Así, el complemento alimenticio elaborado en este estudio, aportó mejoras significativas en los parámetros evaluados, indicando el potencial del producto como promotor de efectos beneficiosos sobre el estado de salud física, así como sobre el estado inmunológico del organismo. 
Palabras clave: Suplementación; Bifidobacterium lactis; Inmunomodulación; Desarrollo de productos.

\section{Introdução}

A alimentação é fator primordial tanto na prevenção quanto na promoção da saúde humana, o que possibilita o controle de várias Doenças Crônicas Não Transmissíveis (DCNTs), como diabetes, hipertensão, neoplasias e insuficiência cardíaca (Raizel et al., 2011). O papel da alimentação equilibrada na manutenção da saúde e na prevenção de doenças vem despertando o interesse da comunidade científica.

Atualmente, os consumidores têm se mostrado mais conscientes e preocupados com seu estilo de vida. Isso aumentou a demanda por alimentos promotores de saúde e bem-estar, com propriedades funcionais (Martins et al., 2013), colaborando, assim, para o desenvolvimento de novos produtos enriquecidos com componentes fisiologicamente ativos (Alves et al., 2008). Neste contexto, surgem os suplementos alimentares, que são preparações destinadas a fornecer nutrientes bioativos como proteínas, aminoácidos, fibras, vitaminas, minerais e ácidos graxos, visando atender a demanda destes no organismo (Bacurau, 2007). No mercado, existem variadas formulações de suplementos alimentares, porém, não é comum encontrar suplementos alimentares com incorporação de probióticos. Além disso, um suplemento que contenha proteínas do soro de leite, cujos efeitos são bem estabelecidos e $\beta$-glucanas, com seu potencial imunomodulador sobre o hospedeiro, é uma novidade a ser investigada.

Alguns estudos estão focados em indivíduos idosos e nas estratégias para aumentar a massa muscular dessa parcela da população. Com o avanço da idade, ocorre naturalmente um processo denominado sarcopenia (perda de massa muscular) (Tieghi, 2014). Com intuito de reduzir este processo, o uso de suplementos a base de proteínas e aminoácidos, cuja principal função é a reparação e reconstrução dos tecidos, aliados ao exercício físico, vêm sendo testado (Tieghi, 2014).

Neste contexto, as proteínas solúveis do soro de leite possuem peptídeos bioativos de boa digestibilidade, especialmente os de cadeia ramificada (Oliveira; Bravo; Tonial, 2012). Estes peptídeos estão relacionados a fatores de crescimento, reconstrução e reparação de músculos e ossos, geram energia, proteção ao sistema cardiovascular, apresentam atividade antimicrobiana, antiviral e possuem atividade imunoestimulante (Haraguchi; Abreu; Paula, 2006; Oliveira; Bravo; Tomial, 2012; Renhe, 2008; Sousa et al., 2012; Sgarbieri, 2005).

Entre os microrganismos com ação probiótica, o Bifidobacterium lactis HN019, 
amplamente consumido incorporado à fermentados lácteos, tem sido relacionado à promoção de efeitos benéficos sobre o metabolismo do hospedeiro, incluindo diminuição dos níveis de LDL (Low Density Lipoproteins) (Bernini et al, 2016; Ejtahed et al., 2011), aumento do colesterol HDL (High Density Lipoproteins) em mulheres adultas, (Sadrzadeh-Yeganeh et al., 2010), melhor tolerância à glicose durante a gravidez (Laitinen; Poussa; Isolauri, 2009; Luoto et al., 2010), melhora em biomarcadores inflamatórios e oxidativos em indivíduos saudáveis e pacientes com síndrome metabólica (Bernini et al., 2018).

Já as $\beta$-glucanas, obtidas da parede celular de Saccharomyces cerevisiae, são polissacarídeos estruturais destes organismos, bem como de outros fungos, bactérias e alguns cereais. Estes compostos têm demonstrado efeitos benéficos na saúde humana, especialmente como um modificador da resposta biológica. Ao ser reconhecida pelo organismo, desencadeia-se uma série de eventos positivos na resposta imune. Também apresenta atividades antitumoral, anti-inflamatória, antimutagênica, hipocolesterolêmica e hipoglicêmica (Magnani; Castro-Gómez, 2009). Por outro lado, seu consumo, por suas propriedades antigenotóxicas, pode inclusive prevenir danos ao DNA, que estão associadas a doenças relacionadas a danos oxidativos, como o câncer (Magnani et al., 2011).

Dessa forma, a elaboração de um suplemento alimentar que, além das propriedades funcionais das proteínas do soro de leite, possa ser veículo para microrganismos probióticos e $\beta$-glucana, apresenta-se como uma boa alternativa para o mercado de suplementos alimentares. O objetivo do presente trabalho foi elaborar um suplemento alimentar, com propriedades funcionais, à base de isolado proteico de soro de leite, probiótico e $\beta$-glucana, determinar a composição centesimal do produto, sua estabilidade durante o armazenamento e verificar os efeitos do produto na composição corporal e imunidade de mulheres fisicamente ativas.

\section{Material e Métodos}

Trata-se de um estudo quantitativo, pois utilizou a coleta de dados numéricos que foram analisados aplicando análises estatísticas pertinentes ao estudo (Pereira et al., 2018).

\subsection{Cultivo de Bifidobacterium animalis spp. lactis HN019}

Foi utilizada a cultura comercial Bifidobacterium animalis spp. lactis HN019 Howaru ${ }^{\circledR}$ Bifido (Dupont) ressuspensa em $100 \mathrm{~mL}$ de leite em pó desnatado reconstituído a 
$10 \%(\mathrm{~m} / \mathrm{v})$ previamente esterilizado a $115^{\circ} \mathrm{C} / 10 \mathrm{~min}$. A cultura foi incubada a $37^{\circ} \mathrm{C} / 24 \mathrm{~h}$. Foram incorporados $100 \mathrm{~g}$ de isolado proteico de soro de leite em pó $(90 \%$ (m/m) de proteína) (Alpha Galvano Quimica Bras. Ltda, São Paulo, Brasil) a cada 100 mL do leite fermentado (1:1). A mistura obtida foi liofilizada e moída mecanicamente até obtenção de um pó, que foi mantido sob refrigeração $\left(4^{\circ} \mathrm{C}\right)$ até o uso.

\subsection{Obtenção da $\beta$-Glucana}

A $\beta$-Glucana foi extraída da parede celular da levedura comercial Saccharomyces cereviseae utilizada em panificação. A autólise da levedura foi realizada de acordo com o procedimento de Darpossolo (2010).

\subsection{Obtenção de leite em pó delactosado}

Leite em pó desnatado foi reconstituído a $50 \%(\mathrm{~m} / \mathrm{v})$. Foi adicionada $\beta$-galactosidase $0,02 \mathrm{~mL} / \mathrm{g}$ de lactose presente na solução de leite (conforme recomendação do fabricante). Após homogeneização, a solução foi hidrolisada a $7^{\circ} \mathrm{C} / 24 \mathrm{~h}$ e em seguida liofilizada. O leite liofilizado foi moído e armazenado a $4^{\circ} \mathrm{C}$ até uso. $\mathrm{O}$ grau de hidrólise do leite foi obtido determinando a concentração de lactose antes da hidrólise (inicial) e depois da hidrólise (final), utilizando o método da Lactose-Metilamina (Nickerson; Vujicic; Lin, 1975).

\subsection{Suplemento alimentar}

O suplemento alimentar foi elaborado pela mistura dos ingredientes: isolado proteico de soro de leite (80,75\%), Bifidobacterium animalis spp. lactis HNO19 (3,49\%), açúcar (8,08\%), cacau em pó $100 \%(3,23 \%)$, $\beta$-glucana $(0,40 \%)$ e leite em pó delactosado $(4,05 \%)$ (CastroGomez; Belloli, 2019). Após a homogeneização, o produto foi acondicionado em potes plásticos (300 g), contendo um dosador plástico com capacidade de $20 \mathrm{~g}$ do suplemento alimentar.

O produto foi avaliado quanto a sua composição centesimal, segundo metodologias propostas pelo Instituto Adolfo Lutz (2008). As análises foram realizadas em triplicata. A contagem de B. lactis HN019 foi realizada de acordo com Silva et al. (2020). Análise microbiológica de Salmonella sp, Escherichia coli e Staphylococcus aureus foram realizadas para garantir a segurança dos participantes do Estudo com humanos (Brasil, 2001). O produto 
foi avaliado logo após a fabricação e aos 30 e 60 dias após a fabricação

\subsection{Estudo com humanos}

O estudo foi aprovado pelo Comitê de Ética da Universidade Pitágoras Unopar (Londrina - PR). Foram recrutadas 34 mulheres com idade entre 55 e 68 anos de idade. Foram critérios de inclusão idade entre 55 e 70 anos, praticar atividade física três (3) vezes/semana e estar disposta a consumir o suplemento alimentar diariamente por 90 dias. As voluntárias praticavam os exercícios físicos com intensidade, repetições e tempo semelhantes. Voluntárias do estudo relataram algum grau de intolerância à lactose, desta forma, o produto obtido foi delactosado. As voluntárias foram recrutadas em uma academia localizada em Bento Gonçalves - RS, e após tirarem dúvidas, aceitaram participar da pesquisa e assinaram o Termo de Consentimento Livre e Esclarecido. Entre as recrutadas, 24 foram alocadas no grupo que recebeu o suplemento alimentar, enquanto 10 voluntárias não consumiram o suplemento. O grupo de voluntárias foi avaliado quanto a concentração de imunoglobulina A salivar e dados antropométricos.

As voluntárias mantiveram seus hábitos alimentares regulares, exceto pela solicitação de excluirem o consumo de probióticos.

O suplemento alimentar foi consumido diariamente por 90 dias, em uma dose de $20 \mathrm{~g}$. Nos dias que praticavam atividade física, o suplemento alimentar era consumido logo após o treino. As voluntárias receberam potes contendo $300 \mathrm{~g}$ de produto a cada 15 dias.

A concentração de IgA foi determinada utilizando-se kit ELISA (E80-102, Bethyl Laboratories, Montgomery, EUA).

Também foram realizadas medidas antropométricas, avaliando massa gorda e massa magra corporal. A estatura de cada voluntária foi avaliada por meio de um estadiômetro (Embreex, Brusque, Brasil), enquanto o peso corporal por balança (A150, Brião, Cachoeira do Sul, Brasil). As medidas das dobras cutâneas foram obtidas com adipômetro (Cescorf, Porto Alegre, Brasil), com molas de $10 \mathrm{~g} / \mathrm{mm}^{2}$, e a circunferência corporal foi medida com fita antropométrica (Cescorf, Porto Alegre, Brasil). Massa gorda e massa magra corporal foram avaliados de acordo com os métodos descritos por Jackson e Pollock (1978). As medidas antropométricas foram realizadas três vezes durante os 90 dias anteriores ao consumo do suplemento alimentar, uma vez a cada mês, e três vezes durante os 90 dias do consumo, uma vez a cada mês. 


\subsection{Análise estatística}

Os resultados foram submetidos à análise de variância (ANOVA) e teste de Tukey utilizando o programa estatístico PAST e nível de significância de 5\% (p<0,05). Foram comparados os efeitos nos parâmetros analisados antes, durante e após o uso do suplemento.

\section{Resultados e Discussão}

O suplemento alimentar elaborado utilizando isolado proteico de soro de leite delactosado, Bifidobacterium lactis HN019 e $\beta$-glucana apresentou alta concentração de proteína e baixo teor de gordura, conforme observado na Tabela 1. De acordo com a Portaria $n^{\circ} 27$, de 13 de janeiro de 1998 (Brasil, 1998a), que trata do Regulamento técnico referente à informação nutricional complementar, um produto será considerado como fonte de proteínas se tiver no mínimo 20\% da Ingestão Diária Recomendada (IDR) de referência por 100 g de alimentos sólidos ou $5 \%$ da IDR por $100 \mathrm{~mL}$ de alimentos líquidos. O produto será considerado como alto teor se apresentar no mínimo 10\% da IDR por $100 \mathrm{~mL}$ (líquidos). Para ser considerado suplemento proteico, o produto deve apresentar no mínimo $10 \mathrm{~g}$ de proteína na porção consumida (Brasil, 2010). Desta forma, o suplemento alimentar elaborado se enquadra como produto com alto teor de proteínas e como suplemento proteico, fornecendo 13,8 g de proteína por porção. Quanto ao teor de lactose do leite, antes da adição da $\beta$ galactosidase, era de 4,9 g/100 g. Após ação da enzima a $7^{\circ} \mathrm{C} / 24 \mathrm{~h}$, o teor de lactose foi de 0,4 g/100 g, com hidrólise de 91,8\%. De acordo com a Portaria $\mathrm{n}^{\mathrm{o}} 29$ (Brasil, 1998b), os alimentos especialmente formulados para atender portadores de intolerância à lactose podem conter no máximo $0,5 \mathrm{~g}$ a cada $100 \mathrm{~g}$ ou $\mathrm{mL}$ do produto final. Assim, o suplemento alimentar elaborado atende a legislação vigente e pode ser classificado como produto delactosado ou sem lactose. 
Tabela 1: Composição centesimal do suplemento alimentar elaborado isolado proteico de soro de leite delactosado, Bifidobacterium lactis HN019 e $\beta$-glucana.

\begin{tabular}{lc}
\hline Componente & Média \pm Desvio Padrão \\
\hline Proteínas (g/100g) & $68,90 \pm 0,08$ \\
Carboidrato (g/100g) & $21,60 \pm 0,07$ \\
Umidade (g/100g) & $6,00 \pm 0,21$ \\
Cinzas (g/100g) & $2,70 \pm 0,10$ \\
Gordura (g/100g) & $0,80 \pm 0,05$ \\
\hline
\end{tabular}

Fonte: Autores.

A recomendação do Ministério da Saúde indica que, para indivíduos saudáveis, uma dieta balanceada deve conter $2000 \mathrm{Kcal} / \mathrm{dia}$, (Brasil, 2008). Os valores diários recomendados para ingestão de proteínas, carboidratos e lipídeos devem ser de 75 g/dia, 300 g/dia e 55 g/dia, respectivamente. Levando-se em consideração estas recomendações, o consumo de uma porção diária de $20 \mathrm{~g}$ do suplemento alimentar desenvolvido fornece $18 \%$ da ingestão diária de proteínas, $1,5 \%$ de carboidratos e $1,5 \%$ da ingestão diária de lipídeos.

O suplemento alimentar desenvolvido apresentou altas contagens do probiótico, conforme apresentado na Tabela 2. Embora a legislação brasileira (Brasil, 2018) ou órgãos reguladores internacionais, como a FAO (FAO/WHO, 2001) não determinem a quantidade de células probióticas adequadas para promoção de efeitos benéficos, muitos estudos recomendam a ingestão diária de pelo menos $10^{8}$ a $10^{9} \mathrm{UFC} /$ porção do produto (Bernini et al., 2016; Granato et al., 2010; Shah, 2000). Além disso, os efeitos atribuídos à probióticos são linhagem específica, de forma que estudos com uma espécie ou linhagem não podem ser extrapolados para outras, mesmo aquelas estreitamente relacionadas (Bertazzoni, et al. 2013; FAO/WHO, 2002; Gueimonde; Salminen, 2006; Hill et al., 2014). O que implica que os efeitos atribuídos à uma linhagem devem ser estudados considerando a estabilidade do probiótico, a matriz no qual foi inserido, bem como a população avaliada. 
Tabela 2: Contagem de Bifidobacterium lactis HN019 no suplemento alimentar durante o período de armazenamento.

\begin{tabular}{lcc}
\hline Período & Contagem (UFC/g) & Log (UFC/g) \\
\hline Fabricação & $6,6 \times 10^{8}$ & 8,8 \\
30 dias de armazenamento & $1,8 \times 10^{8}$ & 8,2 \\
60 dias de armazenamento & $1,0 \times 10^{8}$ & 8,0 \\
\hline
\end{tabular}

Fonte: Autores.

Os resultados das análises antropométricas são apresentados na Tabela 3. As voluntárias da pesquisa apresentaram redução $(\mathrm{p}<0,05)$ nas medidas da supra ilíaca, abdômen, porcentagem de gordura corporal e circunferência abdominal após 60 dias do uso do suplemento alimentar. Ao final dos 90 dias de uso, também observou-se redução $(\mathrm{p}<0,05)$ na massa gorda das voluntárias.

Tabela 3: Avaliação antropométrica de voluntárias praticantes de atividades físicas, com idade entre 55 e 68 anos, antes, durante e após o uso do suplemento alimentar.

\begin{tabular}{lccc}
\hline Medida & Antes do uso & $\begin{array}{c}\text { Durante o uso (60 } \\
\text { dias) }\end{array}$ & $\begin{array}{c}\text { Após o uso (90 } \\
\text { dias) }\end{array}$ \\
\hline Supra ilíaca (mm) & $26,85 \pm 7,55^{\mathrm{a}}$ & $25,54 \pm 7,52^{\mathrm{b}}$ & $25,77 \pm 7,17^{\mathrm{b}}$ \\
Abdômen (mm) & $29,25 \pm 8,18^{\mathrm{a}}$ & $28,06 \pm 8,05^{\mathrm{b}}$ & $28,19 \pm 7,42^{\mathrm{b}}$ \\
Tórax (cm) & $100,21 \pm 9,86^{\mathrm{a}}$ & $99,58 \pm 10,01^{\mathrm{a}}$ & $99,73 \pm 9,99^{\mathrm{a}}$ \\
Cintura (cm) & $81,00 \pm 10,12^{\mathrm{a}}$ & $80,35 \pm 10,76^{\mathrm{a}}$ & $80,58 \pm 11,00^{\mathrm{a}}$ \\
Quadril (cm) & $100,73 \pm 7,71^{\mathrm{a}}$ & $100,15 \pm 7,86^{\mathrm{a}}$ & $100,02 \pm 7,81^{\mathrm{a}}$ \\
Gordura (\%) & $30,63 \pm 4,98^{\mathrm{a}}$ & $29,59 \pm 5,41^{\mathrm{b}}$ & $29,54 \pm 5,61^{\mathrm{b}}$ \\
Massa magra (Kg) & $46,60 \pm 6,23^{\mathrm{a}}$ & $46,54 \pm 6,12^{\mathrm{a}}$ & $46,55 \pm 6,08^{\mathrm{a}}$ \\
Massa gorda (Kg) & $20,98 \pm 6,66^{\mathrm{a}}$ & $20,66 \pm 6,76^{\mathrm{a}}$ & $19,81 \pm 6,84^{\mathrm{b}}$ \\
Circunferência abdominal (cm) & $92,79 \pm 9,27^{\mathrm{a}}$ & $91,71 \pm 9,18^{\mathrm{b}}$ & $91,52 \pm 9,28^{\mathrm{b}}$ \\
\hline
\end{tabular}

Os dados são apresentados como média \pm desvio padrão.

${ }^{a, b}$ Letras diferentes sobrescritas na mesma linha indicam diferença significativa pelo Teste de Tukey $(\mathrm{p}<0,05)$.

Fonte: Autores.

O consumo do suplemento alimentar pelas voluntárias fisicamente ativas trouxe benefícios às mesmas. Tipton et al. (2004) e Toba et al. (2001) também observaram benefícios aos participantes de suas pesquisas. Os autores constataram que os indivíduos que 
ingeriram suplementação com proteína do soro do leite (whey protein), tiveram resultados satisfatórios nas variáveis redução da gordura corporal e aumento da massa magra corporal. Hall et al. (2003) estudaram os efeitos das proteínas do soro de leite sobre a redução de gordura corporal em homens adultos. Os autores observaram que as proteínas do soro interferiram positivamente na redução de gordura em função do alto teor de cálcio e consequentemente, pela ação deste sobre o hormônio 1,25 (OH) 2D. Em estudo realizado por Haraguchi; Abreu; Paula (2006), os autores sugeriram que o consumo de proteína do soro de leite proporcionou redução do percentual de gordura, semelhante ao presente estudo. Os autores mencionam que a redução da gordura corporal ocorre por meio de mecanismos associados ao cálcio, e por apresentar altas concentrações de aminoácidos de cadeia ramificada. Outros estudos também apontaram que o consumo de proteínas do soro de leite pode ser benéfico, já que este possui alto valor nutricional, podendo auxiliar no anabolismo muscular (Tipton et al., 2004), ajuda no controle de perda de massa óssea (Aoe et al., 2001; Toba et al., 2001), regulação da saciedade (Hall et al., 2003), redução da gordura corporal, melhora do desempenho físico, estimulação do sistema imunológico, entre outros, (Haraguchi; Abreu; Paula, 2006; Sgarbieri, 2004). Silva e Mura (2011) relatam que o consumo alimentar insuficiente em energia, e consequentemente, de macro e micronutrientes, pode levar o organismo a situações de estresse, promovendo perda de massa muscular, maior suscetibilidade a doenças infecciosas e baixa imunidade. Assim, o uso de suplementos tornase uma alternativa para minimizar tais situações.

A suplementação das participantes da pesquisa, aliada a prática de atividades físicas, apresentou benefícios ao sistema imunomodulador quando comparado os valores de IgA salivar antes, durante e após o uso do suplemento alimentar (Tabela 4). 
Tabela 4: Níveis de imunoglobulina A coletado de saliva de voluntárias praticantes de atividades físicas, com idade entre 55 e 68 anos, antes, durante e após o uso do suplemento alimentar.

\begin{tabular}{lcc}
\hline \multirow{2}{*}{ Coleta } & \multicolumn{2}{c}{ Consumo do suplemento alimentar } \\
\cline { 2 - 3 } & Sim $(\mathbf{n}=\mathbf{2 4})$ & Não $(\mathbf{n}=\mathbf{1 0})$ \\
\hline Antes do uso & $0,07 \pm 0,05^{\mathrm{b}}$ & $0,06 \pm 0,06^{\mathrm{a}}$ \\
$\mathbf{7}$ dias de uso & $0,09 \pm 0,05^{\mathrm{a}}$ & $0,05 \pm 0,03^{\mathrm{a}}$ \\
$\mathbf{3 0}$ dias de uso & $0,08 \pm 0,05^{\mathrm{a}}$ & $0,06 \pm 0,03^{\mathrm{a}}$ \\
$\mathbf{9 0}$ dias de uso & $0,09 \pm 0,04^{\mathrm{a}}$ & \\
$\mathbf{7}$ dias após suspensão do uso & $0,06 \pm 0,04^{\mathrm{b}}$ & \\
\hline
\end{tabular}

Os dados são apresentados como média \pm desvio padrão.

${ }^{\mathrm{a}, \mathrm{b}}$ Letras diferentes sobrescritas na mesma coluna indicam diferenças significativas pelo Teste de Tukey $(\mathrm{p}<0,05)$.

Fonte: Do autor.

Observaram-se diferenças significativas $(\mathrm{p}<0,05)$ nos níveis de imunoglobulina A na saliva das voluntárias que utilizaram o suplemento alimentar antes, durante e após o uso do suplemento alimentar. O grupo que não recebeu o suplemento alimentar, mas praticou atividade física nas mesmas condições do grupo suplementado, não apresentou diferença significativa $(\mathrm{p}>0,05)$ nos valores de IgA salivar ao longo do tempo. Isto indica que a prática de atividade física não foi a responsável pelo aumento dos níveis de IgA. Desta forma, o consumo de suplemento alimentar contendo proteínas do soro do leite, probiótico e $\beta$-glucana promoveu atividade imunomoduladora nas voluntárias.

Outros estudos não observaram alterações na $\operatorname{IgA}$ salivar frente a exercícios de moderada intensidade (Housh et al., 1991), ao encontro dos resultados encontrados neste estudo. Há ainda escassez de trabalhos que consideram a resposta da IgA durante o exercício, em esportes coletivos ou em situação real de competição. A razão e o modo pelo qual o sistema imune responde aos variados estímulos do exercício não estão completamente esclarecidos (Calder; Kew, 2002).

Um dos componentes do suplemento alimentar elaborado é o B. lactis HN019, que apresenta efeitos sobre vários aspectos da resposta imune natural e adquirida, incluindo a estimulação da resposta não-específica (IgA) e específica (anticorpos) (Gilliland, 2001).

O suplemento alimentar desenvolvido, além de ser bem aceito pelas participantes, apresentou redução em variáveis antropométricas analisadas e aumento dos níveis de imunoglobulina A, proporcionando melhoria no funcionamento do sistema imunológico. As 
participantes não apresentaram indisposição ou alergia aos componentes do produto, mesmo com a maior parte do grupo apresentando intolerância à lactose. Assim, amplia-se o público que pode fazer uso deste suplemento alimentar, atendendo as necessidades de uma parcela da população que apresenta alguma restrição e aquelas que buscam cada vez mais um estilo de vida saudável e maior qualidade de vida.

\section{Considerações Finais}

O consumo do suplemento alimentar contendo proteínas do soro do leite, Bifidobacterium lactis HN019 e $\beta$-glucana durante 90 dias por mulheres com idade entre 55 e 68 anos de idade aliada à prática de atividade física apresentou benefícios a saúde, como redução de medidas antropométricas e aumento nos níveis de imunoglobulina $\mathrm{A}$. $\mathrm{O}$ suplemento alimentar desenvolvido para este estudo foi muito bem aceito pelas participantes e nenhuma delas apresentou indisposição ou alergia aos componentes do produto.

Novos estudos utilizando este suplemento podem ser realizados com grupos específicos, como por exemplo pacientes sob tratamento contra o câncer, ou que apresentam doenças degenerativas. Uma vez que o produto apresenta atividade imunomoduladora, pode se tornar um importante componente do tratamento.

\section{Agradecimentos}

O presente trabalho foi realizado com apoio da Coordenação de Aperfeiçoamento de Pessoal de Nível Superior - Brasil (CAPES) - Código de Financiamento 001.

\section{Referências}

Alves, L. L. et al. (2008). Avaliação sensorial de cream cheeses potencialmente simbióticos utilizando a metodologia de superfície de resposta. Alimentos e Nutrição, Marília, 19(4), 40916

Aoe, S. et al. (2001). Controlled trial of the effects of milk basic protein (MBP) supplementation on bone metabolism in healthy adult women. Bioscience, Biotechnology, and Biochemistry, Abingdon, 65(4), 913-18. https://doi.org/10.1007/s00198-005-2012-3 
Bacurau, R. F. (2007). Nutrição e Suplementação Desportiva. 5. ed. São Paulo: Phorte.

Bernini, L.J. et al. (2016). Beneficial effects of Bifidobacterium lactis on lipid profile and cytokines in patients with metabolic syndrome: A randomized trial. Effects of probiotics on metabolic syndrome. Nutrition, 32(6), 716-19. https://doi.org/10.1016/j.nut.2015.11.001

Bernini, L.J. et al. (2018). Effect of Bifidobacterium lactis HN019 on inflammatory markers and oxidative stress in subjects with and without the metabolic syndrome. British Journal of Nutrition, 120(6), 645-52. https://doi.org/10.1017/S0007114518001861

Bertazzoni, E. et al. (2013). Probiotics and clinical effects: is the number what counts? Journal of Chemotherapy, 25, 193-213. https://doi.org/10.1179/1973947813Y.0000000078

Brasil. (1998a). Ministério da Saúde. Secretaria de Vigilância Sanitária. Portaria no 27, de 13 de janeiro de 1998. Regulamento Técnico referente à Informação Nutricional complementar.

Brasil. (1998b). Ministério da Saúde. Agência Nacional de Vigilância Sanitária. Portaria Portaria $\mathrm{n}^{\circ}$ 29, de 13 de janeiro de 1998. Regulamento técnico para fixação de identidade e qualidade de alimentos para fins especiais.

Brasil. (2001). Ministério da Saúde. Agência Nacional de Vigilância Sanitária - ANVISA. Resolução RDC $\mathrm{n}^{\circ}$ 12, de 02 de janeiro de 2001. Regulamento técnico sobre padrões microbiológicos para alimentos.

Brasil. (2008). Ministério da Saúde. Agência Nacional de Vigilância Sanitária - ANVISA. Guia alimentar para a população brasileira: promovendo a alimentação saudável. Normas e Manuais Técnicos - Série A. Brasília. p.210.

Brasil. (2010). Ministério da Saúde. Agência Nacional de Vigilância Sanitária - ANVISA. Resolução RDC n 18, de 27 de abril de 2010. Regulamento Técnico sobre Alimentos para Atletas. 
Brasil. (2018). Ministério da Agricultura, Pecuária e Abastecimento. Instrução Normativa $n^{\circ}$ No 241, de 26 de julho de 2018. Resolução dispõe sobre os requisitos para comprovação da segurança e dos benefícios à saúde dos probióticos para uso em alimentos.

Calder, C. P. \& Kew, S. (2002). The immune system: a target for functional foods? British Journal of Nutrition. Wallingford. 88(2), 165-77. https://doi.org/10.1079/BJN2002682

Castro-Gomez, R. J. H. \& Belloli, O.B. Composição alimentar a base de proteinas isoladas de soro de queijo, probiotico e betaglucana de levedura e processo para obtenção. Depositante: Universidade Pitagoras Unopar/Instituto Federal de Educação, Ciência e Tecnologia do Rio Grande do Sul - IFRS. Procurador: Cristiane Rumika Minowa. BR 1020180010344. Depósito: 18 jan. 2018. Concessão: 30 jul. 2019.

Darpossolo, F. P. B. et al. (2010). Avaliação do potencial imunoestimulante da Carboximetilglucana de Saccharomyces cerevisiae em frangos de corte (Gallus domesticus). Seminas: Ciências Agrárias, Londrina, 31(1), 231-40.

Ejtahed, H. S. et al. (2011). Effect of probiotic yogurt containing Lactobacillus acidophilus and Bifidobacterium lactis on lipid profile in individuals with type 2 diabetes mellitus. Journal of Dairy Science, South Oak Street, 94(7). 3288-94. https://doi.org/10.3168/jds.20104128

FAO/WHO. (2001). Food and Agriculture Organization of United Nations; World Health Organization. Food and Nutrition paper 85. Probiotics in food - Health and nutritional properties and guidelines for evaluation. (2006). Report of a Joint FAO-WHO expert consultation on evaluation of health and nutritional properties of probiotics in food including powder milk with live lactic acid bacteria. Cordoba, Argentina.

FAO/WHO. (2002). Food and Agriculture Organization of United Nations; World Health Organization. Report of a Joint FAO/WHO working group on drafting guidelines for the evaluation of probiotics in food. London Ontario, Canada. 
Granato, D. et al. (2010). Probiotic dairy products as functional foods. Comprehensive Reviews in Food Science and Food Safety, 9(5), 455-70. https://doi.org/10.1111/j.15414337.2010.00120.x

Gueimonde, M. \& Salminen, S. (2006). New methods for selecting and evaluating probiotics. Digestive and Liver Disease, 38, S242-47. https://doi.org/10.1016/S1590-8658(07)60003-6

Gilliland, S. E. (2001). Probiotics and prebiotics. In: MARTH, E. H.; STEELE, J. L. Applied dairy microbiology. New York: Marcel Dekker, p. 327-343.

Hall, W. L. et al. (2003). Casein and whey exert different effects on plasma amino acids profiles, gastrointestinal hormone secretion and appetite. British Journal of Nutrition, Cambridge, 89(2), 239-48. https://doi.org/10.1079/BJN2002760

Haraguchi, F. K.; Abreu, W. C. \& Paula, H. (2006). Proteínas do soro do leite: composição, propriedades nutricionais, aplicações no esporte e benefícios para a saúde humana. Revista Brasileira de Nutrição, Campinas, 19(4), 488. https://doi.org/10.1590/S141552732006000400007

Hill, C. et al. (2014). Expert consensus document: The International Scientific Association for Probiotics and Prebiotics consensus statement on the scope and appropriate use of the term probiotic. Nature Reviews Gastroenterology \& Hepatology, 11, 506-14. https://doi.org/10.1038/nrgastro.2014.66

Housh, T. J. et al. (1991). The effect of exercise at various temperatures on salivary levels of immunoglobulin A. International Journal of Sports and Medicine, Stuttgart, 12, 498-500. https://doi.org/10.1055/s-2007-1024722

Instituto Adolfo Lutz. (2008) Normas Analíticas do Instituto Adolfo Lutz. São Paulo: Instituto Adolfo Lutz. 4.ed.

Isolauri, E; Salminen, S \& Ouwehand, A. C. (2004). Probiotics. Best Practice \& Research Clinical Gastroenterology, Amsterdam, 18(2), 299-313. https://doi.org/10.1016/j.bpg.2003.10.006 
Jackson, A. S., \& Pollock, M. L. (1978). Generalized equations for predicting body density of men. British Journal of Nutrition, 40(3), 497-504. https://doi.org/10.1079/bjn19780152

Laitinen, K.; Poussa, T. \& Isolauri, E. (2009). Nutrition, allergy, mucosal immunology and intestinal microbiota group. Probiotics and dietary counselling contribute to glucose regulation during and after pregnancy: a randomised controlled trial. British Journal of Nutrition, Cambridge, 101(11), 1679-87. https://doi.org/10.1017/S0007114508111461

Luoto, R. et al. (2010). Impact of maternal probiotic-supplemented dietary counselling on pregnancy outcome and prenatal and postnatal growth: a double-blind, placebo controlled study. British Journal of Nutrition, Cambridge, 103(12), 1792-99. https://doi.org/10.1017/S0007114509993898

Magnani, M. \& Castro-Gómez, R. J. H. (2009). $\beta$-glucana from Saccharomyces cerevisiae: constitution, bioactivity and obtaining. Semina: Ciências Agrárias, Londrina, 29(3), 631-50. http://dx.doi.org/10.5433/1679-0359.2008v29n3p631

Magnani, M. et al. (2011). Protective effect of carboxymethyl-glucan (CMG) against DNA damage in patients with advanced prostate cancer. Genetics and Molecular Biology, Ribeirão Preto, 34(1), 131-34. https://doi.org/10.1590/S1415-47572010005000103

Martins, E. M. F. et al. (2013). Products of vegetable origin: A new alternative for the consumption of probiotic bacteria. Food Research International, 51(2), 764-70. https://doi.org/10.1016/j.foodres.2013.01.047

Nickerson, T. A; Vujicic, I. F \& Lin, A. Y. (1975). Colorimetric estimation of lactose and its hydrolytic products. Journal of Dairy Science, Champaign, 59(3), 386-90. https://doi.org/10.3168/jds.S0022-0302(76)84217-8

Oliveira, D. F.; Bravo, C. E. C. \& Tonial, I. B. (2012). Soro de leite: um subproduto valioso. Revista do Instituto de Laticínios Cândido Tostes, Juiz de Fora, 67(385), 64-71. https://doi.org/10.5935/2238-6416.20120025 
Pereira, A.S., Shitsuka, D.M., Parreira, F.J., Shitsuka, R. (2018). Metodologia da pesquisa científica. [e-book]. Santa Maria. Ed. UAB/NTE/UFSM. Available in: https://repositorio.ufsm.br/bitstream/handle/1/15824/Lic_Computacao_MetodologiaPesquisa-Cientifica.pdf?sequence=1. Accessed in: 13 out. 2020.

Raizel, R. et al. (2011). Efeitos do consumo de probióticos, prebióticos e simbióticos para o organismo humano. Revista Ciência \& Saúde, Porto Alegre, 4(2), 66-74. https://doi.org/10.15448/1983-652X.2011.2.8352

Renhe, I. R. T. (2008). O papel do leite na nutrição. Revista do Instituto de Laticínios Cândido Tostes, Juiz de Fora, 63(363), 36-43.

Sadrzadeh-Yeganeh, H. et al. (2010). The effects of probiotic and conventional yoghurt on lipid profile in women. British Journal of Nutrition, Cambridge, 103(12), 83-103. https://doi.org/10.1017/S0007114509993801

Sgarbieri, V. C. (2004). Propriedades fisiológicas-funcionais das proteínas do soro de leite. Revista de Nutrição, Campinas, 17(4), 397-409. https://doi.org/10.1590/S141552732004000400001

Sgarbieri, V. C. (2005). Revisão: propriedades estruturais e físico-químicas das proteínas do leite. Brazilian Journal of Food Technology, Campinas, 8(1), 43-56.

Shah, N. P. (2000). Probiotic Bacteria: Selective Enumeration and Survival in Dairy Foods. Journal of Dairy Science, 83(4), 894-907. https://doi.org/10.3168/jds.S0022-0302(00)749538

Silva, S. M. C. S. \& Mura, J. D. P. (2011). Tratado de alimentação, nutrição e dietoterapia. 2. ed. São Paulo: Roca.

Silva, J. R. et al. (2020). Probiotic gummy candy with xylitol: development and potential inhibition of Streptococcus mutans UA 159. Research, Society and Development, 9(10), e7369108942. https://doi.org/10.33448/rsd-v9i10.8942 
Sousa, G. T. et al. (2012). Dietary whey protein lessens several risk factors for metabolic diseases: a review. Lipids in Health and Disease, London, 10(11), 67. https://doi.org/10.1186/1476-511X-11-67

Tieghi, A. L. (2014). Suplementos alimentares: funcionam, mas não para todos. Espaço Aberto, São Paulo, n. 156.

Tipton, K. D. et al. (2004). Ingestion of casein and whey proteins result in muscle anabolism after resistance exercise. Medicine and Science in Sports and Exercise, Madison, 36(12), 2073-2081. https://doi.org/10.1249/01.mss.0000147582.99810.c5

Toba, Y. et al. (2001). Milk basic protein promotes bone formation and suppresses bone resorption in health adult men. Bioscience, Biotechnology and Biochemistry, Tokyo, 65(6), 1353-1357. https://doi.org/10.1271/bbb.65.1353

\title{
Porcentagem de contribuição de cada autor no manuscrito
}

\author{
Orlando Barbieri Belloli - 50\% \\ Joice Sifuentes dos Santos - 15\% \\ José Renato Silva - 10\% \\ Ivana Stefanini Carreiro - 5\% \\ Giselle Nobre Costa - 5\% \\ Raul Jorge Hernan Castro-Gomez - 15\%
}

